\title{
Myelopathy Caused by Spinal Dural Arterio-Venous Fistula after First Lumbar Vertebral Body Fracture - A Case Report -
}

\author{
Jin-Woo Kang, M.D., Jung-Hoi Koo, M.D., Dong-Kyu Kim, M.D., \\ Young-Jin Joo, M.D., Tae-Hoon Kim, M.D., Sun-Hong Song, M.D. \\ Department of Rehabilitation Medicine, Gangneung Asan Medical Center, \\ University of Ulsan College of Medicine, Gangneung 210-711, Korea
}

Spinal dural arteriovenous fistula is a rare vascular lesion of the spinal cord associated with progressive myelopathy. Symptoms include progressive gait dysfunction, weakness, sensory loss, and bowel and bladder dysfunction. Because these symptoms overlap with other common causes of myelopathy and the disease is rare, spinal dural arteriovenous fistula is often not suspected and the time to diagnosis is long. We report the case of a 60 -year-old woman who presented with progressive lower limb weakness and gait disturbance diagnosed as spinal dural arteriovenous fistula involving a fractured L1 vertebral body.

Key Words Spinal dural arteriovenous fistula, Myelopathy

\section{INTRODUCTION}

The spinal dural arteriovenous fistula is a rare spinal vessel disease that occurs mostly in middle aged males. ${ }^{1}$ The disease can cause myelopathy by generating highblood pressure of the spinal artery and edema around the

Received August 27, 2010; Accepted October 19, 2010

Corresponding author: Jung-Hoi Koo

Department of Rehabilitation Medicine, Gangneung Asan Medical Center, University of Ulsan College of Medicine, 415, Bangdong-ri, Sacheon-myeon, Gangneung 210-711, Korea

Tel: +82-33-610-4951, Fax: +82-33-610-4960, E-mail: mdjhkoo@gnah. co.kr

(c) This is an open-access article distributed under the terms of the Creative Commons Attribution Non-Commercial License (http:// creativecommons.org/licenses/by-nc/3.0) which permits unrestricted noncommercial use, distribution, and reproduction in any medium, provided the original work is properly cited.

Copyright (c) 2011 by Korean Academy of Rehabilitation Medicine spine, resulting in gradual gait disturbance, weakness of the lower extremities, impairment of sensory function, abnormal defecation, impaired urinary function, and sexual dysfunction. ${ }^{2}$

Since the symptoms are not different from those of more common spinal diseases like spinal stenosis, herniation of intervertebral disc, ossification of ligament and spinal fracture, accurate diagnosis can be difficult. Even image examination can result in obscure or normal findings, which necessitates caution in the diagnosis process. If prompt and appropriate diagnosis can be achieved, symptom improvement is possible by spinal arterial embolization or other operations. Therefore, the understanding of this rare but treatable disease is critical for physicians who manage and treat myelopathy. We report on a patient with progressive myelopathy due to a first lumbar vertebral body bursting fracture followed by 
spinal dural arteriovenous fistula, and provide a literature review. ${ }^{1,3}$

\section{CASE REPORT}

A 60-year-old female patient was admitted to the inpatient treatment section from the outpatient therapy wing of the physical medicine and rehabilitation department for the evaluation and treatment of impaired walking due to gradual weakening of the bilateral lower extremities that had lasted for three months. The patient seemed to have cognitive impairment, and did not have accurate memory of the trauma. However, according to the report of a family member, the patient had been medicated for cerebral infarction, hypertension, and osteoporosis, with no problems in ambulation and other activities of daily living before slipping down three months ago. After that, she had been in a bed ridden state and showed gait disturbance. Based on neurological examination, her mental status was alert, but her orientation, memory, calculation and other cerebral functions were generally impaired, whereas her speech was intact. In terms of the cerebellar function, there were no specific findings on both of the upper extremities, and the lower extremities could not be checked due to muscle weakness. Although an accurate evaluation of motor function was challenging during the manual muscle test, the upper extremities were found to be in normal range. For the lower extremities, the flexor and extensor of the hip and knee of both sides were in poor grade, and the dorsiflexor and plantarflexor of both ankle joints were degraded to trace grade level. There was no limitation in passive range of motion. In both lower extremities, the spasticity corresponded to the Modified Ashworth Scale I grade. Although accurate evaluation for sensory function was also difficult, the patient showed a relatively intact result in the pinprick test, but the proprioception and vibration sensation were impaired. Deep tendon reflex was normal for both upper extremities but increased in both lower extremities, and Barbinski's signs and ankle clonus were found on both sides. Results of the functional evaluation showed impossibility of independent ambulation. Although the activities of daily living using the upper extremities were possible, the patient needed considerable assistance in other areas. In terms of urinary function, urinary retention was observed, so we inserted a foley catheter since the patient was incapable of self-voiding. In addition, due to severe constipation, medication was provided and we helped defection with the use of glycerine enema, 2-3 per day as needed. The first lumbar vertebral body bursting fracture was found in the simple film taken at admission. From the magnetic resonance imaging (MRI) of the lumbar spine, the spinal dural arteriovenous fistula was found in the first lumbar vertebral body bursting fracture in addition to the shades of dilated vein in the periphery of the spines starting from the fifth thoracic vertebra to the cornus medullaris. As a result, the spinal cord edema was accompanied, and the specific findings of high signal, light shade of the spine were observed from the T2 weighted image (Fig. 1-A). Although electromyography and somatosensory evoked potential test were considered, we could not conduct them because of the patient's transfer to the neurosurgical department due to urgent need of arterial embolization. For more accurate location mapping of the spinal arteriovenous fistula, we conducted spinal vessel angiography and found that the arteriovenous fistula originated from the twelfth intercostal artery and
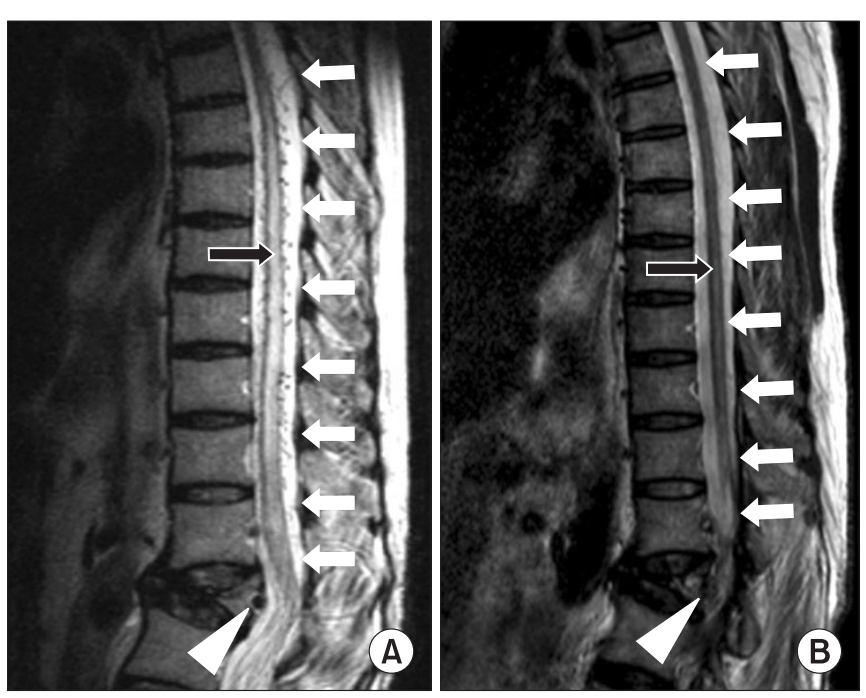

Fig. 1. (A) Sagittal T2-weighted magnetic resonance image of the thoracolumbar spine showing edema of the thoracic cord and conus medullaris (black arrow) and regional dilated perimedullary vessels (white arrows) suggestive of a spinal dural arteriovenous fistula (white arrow head) in the fractured vertebral body at L1 level. (B) After glue embolization, sagittal T2-weighted magnetic resonance image of the thoracolumbar spine showing normal finding of the thoracic cord and conus medullaris (black arrow) and disappearance of dilated perimedullary veins (white arrows) and a spinal dural arteriovenous fistula (white arrow head). 
first lumbar radicular artery. In addition, the spinal vein located on the anterior and posterior spine that continued to the cervical vertebra region was observed (Fig. 2-A). Since the artery of interest was not that large, we planned the embolization procedure employing glue (n-butyl cyanoacrylate) rather than the operation. After embolization of the number twelve right thoracic vertebrate intercostal artery and first lumber, the embolization of all fistula was followed. As a result, there was no venous plexus observed around the spine (Fig. 2-B), and the neurologic symptoms of the patient improved. The hip flexor and knee extensor improved to 'fair grade' and the ankle plantar flexor improved to 'poor grade'. Although we were not able to check the sensory function due to the patient's impaired cognition, big changes were not observed after the treatment. As a result of the post therapy follow-up using MRI to see the condition of spinal cord edema, we found that the spinal vein was dilated due to arteriovenous fistula not being found, as well as the spinal cord edema around the artery. Therefore, we were able to observe normal low signal intensity of the spinal cord from the T2 weighted image (Fig. 1-B). With the help of rehabilitation therapy including strengthening and endurance exercise of the lower extremities, gait training, and functional electrical
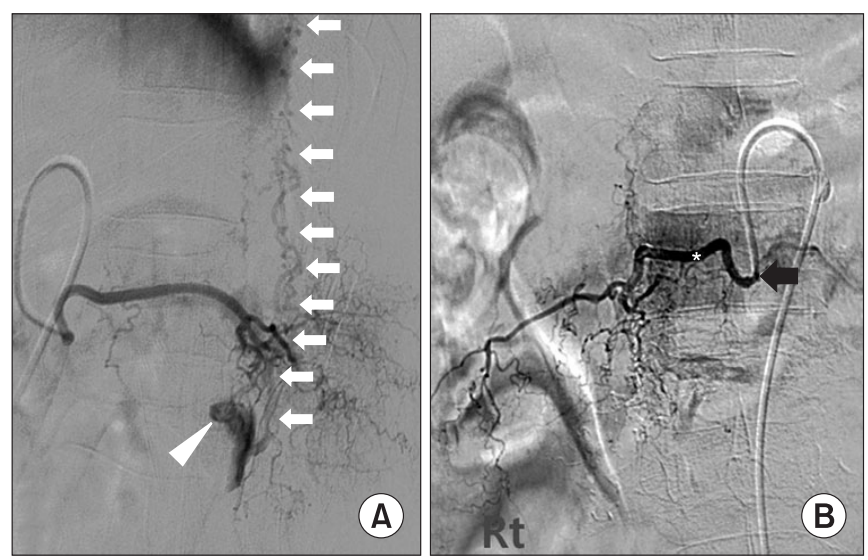

Fig. 2. (A) Selective spinal angiogram of the right $\mathrm{L} 1$ segmental radicular artery showing a fistulous connection (white arrow head) between the feeding artery and the perimedullary venous plexus (white arrows) (The lateral view). (B) Right spinal angiograms following microcatheter placement for glue ${ }^{*}$ ) embolization in the right L1 and T12 radicular artery from above and across to below the fistula (Black arrow). After embolization, there was complete cessation of flow through the fistula (The anteropostetrior view). stimulation to both tibialis anterior, the patient showed gradual improvement and could ambulate about 2-3 m. The urinary symptoms also improved and the patient was able to self-void after removal of the foley catheter following normal functioning results based on the urodynamic study. Bowel function also improved and the patient was capable of daily defecation.

\section{DISCUSSION}

Although spinal dural arteriovenous fistula is a rare disease with unknown incidence, incidences after operations for herniated intervertebral discs or traumalike fractures have been reported. ${ }^{4-6}$

Although the compression or bursting fracture of the spinal cord due to osteoporosis is frequently found by physicians in females undergoing menopause, arteriovenous fistula by fracture developing into myelopathy and paraparesis of the lower extremities is very rare. However, relatively prompt and accurate diagnosis of this condition can generate reversible recovery. In some cases, accurate diagnosis can take over two years due to the difficulty of diagnosis. ${ }^{3}$

The suggested mechanism of spinal dural arteriovenous fistula is spinal cord ischemia due to venous hypertension around the spinal cord, caused by arteriovenous fistula between the arterial and dural spinal venous plexus, followed by high pressured reflux. ${ }^{1,7}$

Magnetic resonance imaging (MRI) is useful in diagnosis, and the $\mathrm{T} 2$ weighted image can observe the spinal cord edema, which shows high signal intensity in the spinal cord as well as the dilated veins around the spinal cord. For more accurate arteriovenous fistula locating, angiography can be used, which is beneficial for combined diagnosis and treatment. ${ }^{1,8}$ In the case of our patient, the arteriovenous fistula located at the posterior center of the first lumbar vertebral body at the bursting fracture of vertebra area was found by angiography, and all the feeding artery were linked to the area. This showed the possibility of formation of the spinal vein and arteriovenous fistula caused by the damage of the spinal radicular artery by fracture.

The symptoms addressed by the patients were not very different from the symptoms reported by other spinal dural arteriovenous fistula patients, and the myelopathic symptoms were not characteristic. It was hard to 
differentially diagnose the illness from other spinal diseases based on the neurological symptom. ${ }^{2}$

Two methods of treatment, including operation and embolization are conducted most frequently for therapy. The treatment principle is very simple and involves lowering of venous hypertension by blocking the arteriovenous fistula. The method appropriate to the condition of the patient is selected, and with the development of intravascular intervention like glue or coil embolization, the embolization is frequently employed as the first line of treatment. Although embolization is used most frequently due to its non-invasiveness and limited complications, when too many feeding arteries are connected to a single vein, the catheter is hard to approach because of the small diameter of the feeding artery. Also, when the anterior spinal artery is connected to the arteriovenous fistula via the feeding artery and has the possibility of causing anterior spinal artery embolism, microsurgery with selective arteriovenous ligation after laminectomy is preferred. ${ }^{9}$ Although the recovery rate and results vary with the patient's pre-treatment myelopathy severity, a majority of patients show similar patterns of improvement to our patient in the radiological evaluation, and improvements in neurological symptoms have been also reported. ${ }^{1,10}$

We conducted MRI because myelopathy was suspected based on the neurological examination of the patient, and were able to diagnose spinal dural arteriovenous fistula, which rarely occurs by the bursting fracture of vertebra. In addition, prompt embolization through consultation with the department of neurosurgery contributed towards better neurological prognosis, and the rehabilitation therapy supported functional recovery. If spinal dural arteriovenous fistula is diagnosed and treated promptly and appropriately, neurological recovery is expected, which is why understanding the disease is important for physicians who manage and treat myelopathy.

\section{REFERENCES}

1. Gilbertson JR, Miller GM, Goldman MS, Marsh WR. Spinal dural arteriovenous fistulas; MR and myelographic findings. AJNR Am J Neuroradiol 1995; 16: 2049-2057

2. Atkinson JL, Miller GM, Krauss WE, Marsh WR, Piepgras DG, Atkinson PP, Brown RD Jr, Lane JI. Clinical and radiographic features of dural arteriovenous fistula, a treatable cause of myelopathy. Mayo Clin Proc 2001; 76: 1120-1130

3. Isu T, Iwasaki Y, Akino M, Koyanagi I, Abe H. Magnetic resonance imaging in cases of spinal dural arteriovenous malformation. Neurosurgery 1989; 24: 919-923

4. Heuer GG, Gabel BC, Bhowmick DA, Stiefel MF, Hurst RW, Schuster JM. Symptomatic high-flow arteriovenous fistula after a C2 fracture: case report. J Neurosurg Spine 2008; 8: 381-384

5. Suh DC, Choi GC, Sung KB, Kim KK, Rhim SC. Spinal osseous epidural arteriovenous fistula with multiple small arterial feeders converging to a round fistular nidus as a target of venous approach. AJNR Am J Neuroradiol 2004; 25: 69-73

6. Leape LL, Palacios E. Acute traumatic vertebral arteriovenous fistula. Ann Surg 1971; 174: 908-910

7. Hui F, Trosselo MP, Meisel HJ, Alvarez H, Sequeira E, Lasjaunias P. Paraspinal arteriovenous shunts in children. Neuroradiology 1994; 36: 69-73

8. Jones BV, Ernst RJ, Tomsick TA, Tew J Jr. Spinal dural arteriovenous fistulas; recognizing the spectrum of magnetic resonance imaging findings. J Spinal Cord Med 1997; 20: 43-48

9. Park SB, Han MH, Jahng TA, Kwon BJ, Chung CK. Spinal dural arteriovenous fistulas; clinical experience with endovascular treatment as a primary therapeutic modality. J Korean Neurosurg Soc 2008; 44: 364-369

10. Nagata S, Morioka T, Natori Y, Matsukado K, Sasaki T, Yamada T. Factors that affect the surgical outcomes of spinal dural arteriovenous fistulas. Surg Neurol 2006; 65: 563-568 